\title{
RESULTS OF ANA TESTING ACCORDING TO MEDICAL REQUESTING SPECIALTIES - A RETROSPECTIVE ANALYSIS OF A LARGE SAMPLE OF PATIENTS REFERRED FOR AUTOANTIBODIES TESTING
}

\author{
Wilton Ferreira Silva Santos ${ }^{1},{ }^{\star}$, Leandro Kegler Nardes ${ }^{2}$, Daniele de Castro Félix ${ }^{1}$, Igor Cabral Santos de Melo ${ }^{3}$ \\ 1.Sabin Medicina Diagnóstica, Brasília (DF), Brazil; 2.Escola Superior de Ciências da Saúde, Brasília (DF), Brazil; 3.Universidade Evangélica \\ de Goiás, Anápolis (GO), Brazil. \\ *Corresponding author: wiltonss@uol.com.br
}

\section{BACKGROUND}

Antinuclear antibody (ANA) test is the most requested exam for the diagnostic evaluation of autoimmune diseases. The number of medical specialties that use ANA in the investigation of organ-specific and non-organ specific autoimmune diseases has increased progressively in recent decades. The objective of this work was to determine the medical specialties that most request ANA and the patterns more frequently associated with the requesting specialties.

\section{METHODS}

Results of ANA tests performed between January and June 2017 were reviewed. The referring medical specialty was identified and the fluorescent patterns associated with the specialties were analyzed. ANA tests were performed by indirect immunofluorescence on HEp-2 cells. Titers $\geq 1 / 80$ were reported as positive. International Consensus on ANA Patterns (ICAP) and the Brazilian Consensus on Autoantibodies (BCA) recommendations were used for patterns classification.

\section{RESULTS}

In the study period, 52,117 samples, in which it was possible to identify the requesting medical specialty, were reviewed. Of this total, the medical specialties that most requested ANA were: general practitioner (20.1\%); dermatology (15\%); gynecology (9.9\%); rheumatology (8.5\%); cardiology (5.8\%); gastroenterology (5.7\%); endocrinology (5.6\%); orthopedics (5.4\%) and others (24\%).In patients referred by rheumatologists $(n=4.226)$, ANA positivity $(35.7 \%)$ was significantly higher than in those referred by the other areas analyzed together $(n=50,564$, positivity $=19.6 \%, p<0.0001)$. Regarding the ANA patterns analysis by cellular domain, the presence of ANA with nuclear pattern varied between specialties from 63.3 (oncology) to 88.1\% ("others") (mean = 84.4\%). The proportion of cytoplasmic patterns ranged from 0.6 (pediatrics) to $11.7 \%$ (oncology) and mitotic patterns from 0.0 (geriatrics) to $3.1 \%$ (pneumology) (means $=4.6$ and $1.6 \%$ respectively). The proportion of sera with mixed ANA patterns (BCA) ranged from 5.0 (cardiology) to $23.3 \%$ (oncology) (mean =9.4\%). Except for sera referred by pneumologists and oncologists, the ICAP patterns more observed in all medical specialties were AC-4 (nuclear fine speckled) and AC-2 (nuclear dense fine speckled). In samples from oncology, AC-4 and mixed patterns were observed in same proportion (23.3\%), with a relative high proportion of cytoplasmic patterns (11.7\%). Samples referred by pneumologists showed AC-4 and BAC-3 (Brazilian anti-cell-3, nuclear quasi-homogeneous) as the most frequent patterns. In samples from rheumatology, AC-4, AC-2, BAC-3, AC-1 (nuclear homogeneous), AC-5 (nuclear coarse speckled) and mixed/ composite patterns were the most prevalent ones. Regarding the analysis of antibody titers, there were a higher frequency of moderate $(1 / 320-1 / 640)(46.4 \%)$ and high ( $\geq 1 / 1280)(10.8 \%)$ titers in patients referred by rheumatologists, when compared to other medical specialties (moderate $=40.9 \%$; high $=4.6 \%)(p<0.0001)$.

\section{CONCLUSION}

Rheumatologists were responsible for less than $10 \%$ of ANA exams requested during the study period. ANA positive results and moderate to high titles of autoantibodies were more frequently observed in patients referred by rheumatologists. AC-4 and AC-2 were the most prevalent ANA patterns observed regardless the requesting specialty. A relative high proportion of mixed and cytoplasmic patterns were observed in patients referred by oncologists and the nuclear BAC-3 in patients referred by pneumologists.

\section{KEYWORDS}

Autoantibodies, Antinuclear Antibodies, ANA testing, Laboratory diagnosis. 